\section{Cureus}

Received 11/11/2013

Review began 11/12/2013

Published 02/10/2014

\section{(c) Copyright 2014}

Weidlich et al. This is an open access article distributed under the terms of the Creative Commons Attribution License CC-BY 3.0., which permits unrestricted use, distribution, and reproduction in any medium, provided the original author and source are credited.

\title{
A Method for Online Dose-Guided Adaptive Radiotherapy
}

\author{
Georg A. Weidlich ${ }^{1}$, Uma Swamy ${ }^{2}$ \\ 1. Radiation Oncology, National Medical Physics and Dosimetry Comp., Inc 2. California Cancer Center, \\ 7257 N. Fresno St., Fresno, CA 93720
}

$\square$ Corresponding author: Georg A. Weidlich, weidlich@hotmail.com

Disclosures can be found in Additional Information at the end of the article

\section{Abstract}

A process was developed to allow the delivery of online dose-guided adaptive radiotherapy (DART). DART was defined as the method to utilize patient imaging data and treatment planning data, such as dose fluences to assess the validity and quality of the treatment and the dose delivered to the target volume and critical anatomical structures of the patient. A system was established to adapt radiotherapy treatment parameters to ensure optimized treatment.

Categories: Radiation Oncology

Keywords: real-time system, adaptive radiotherapy, dose guidance

\section{Introduction}

Historically, adaptive radiation therapy is applied either as a geometry-based or dose-based method. Yan, et al. [1] employed the geometry-based approach and introduced patient-specific target positioning with a margin that was derived from the first several treatment fractions and associated imaging events. A modified margin was then used for replanning of the treatment that accounted for the stochastic variations in target position. Similar geometric as well as dose-based methods addressing set-up errors and organ motion by de la Zerda, Armbruster, and Xing [2], Wu, et al. [3], as well as Yan, et al. [4] were conducted that included replanning and new margin calculations. In these studies, the dose delivered during prior treatment fractions was included in the replanning. Another geometry-based approach was implemented by Court, et al. [5] and Rijkhorst, et al. [6] where the treatment target shape, size, and rotation was investigated and corrected by adapting the multileaf collimator shape and angle accordingly.

Wu, Liang, and Yan [7] developed a concept in which the accumulated dose was calculated and compared with the original treatment plan and any dose deficit was corrected by dose compensation with 3DCRT and IMRT replanning. Such adaptive plan opimization was further investigated and analyzed by Birkner, et al. [8] as well as Lof, Lind, and Brahme [9].

As Ghilezan, Yan, and Martinez [10] have shown in their review article about adaptive radiation therapy for prostate cancer, various approaches to adaptive radiation therapy were employed, focusing on image guidance, immobilization devices, margin definition to escalate dose, and to adapt the treatment delivery to the change in patient anatomy.

While image-guided radiotherapy (IGRT) provides reproducible and precise patient anatomybased positioning prior to the radiotherapy delivery, it does not provide any dosimetric information. $\mathrm{kV}$ or MV conebeam CT (CBCT) are used to verify patient or organ positioning before treatment delivery. Adjustments are made to reproduce locations as presented during 
the original simulation process. As the treatment efficacy and the potential for side-effects are determined by the correct dose delivery, it becomes necessary to use quantitative dose evaluation tools on the day of treatment to determine dosimetric changes due to altered anatomy, i.e., tumor shrinkage, tumor motion, weight gain, or weight loss. This information will be used to adapt treatment parameters accordingly. Such tools are presently not commercially available.

The goal of this research project was the development of a comprehensive method and process for dose-guided adaptive radiotherapy (DART). This method allows the clinical user to utilize imaging information of the patient on the day of treatment in the treatment position, combined with the previously acquired IMRT dose fluence matrix to predict quantitatively the dose to be delivered on the day of treatment. The resulting dose distribution was compared to the dose distribution from the treatment plan and deviations were calculated in a $1 \mathrm{D}, 2 \mathrm{D}$, and volumetric format. Dose-volume-histograms (DVH) were calculated and compared with the treatment plan DVH for target volumes and critical structures. A method of parameters were developed to either accept the treatment as is or make the necessary adaptations to the treatment plan to ensure that planned dose to the target and critical structures will be delivered as planned.

\section{Materials And Methods}

The criteria for the selection of patients is based on the following considerations:

a.) anticipated tumor shrinkage during the course of treatment

b.) anticipated weight loss or weight gain during the course of treatment

c.) nearby critical anatomical strucures embedded in the PTV

d.) prolonged treatment course

e.) hypo-fractionated treatment course, i.e., stereotactic or accelerated partial breast irradiation

The process of dose-guided adaptive radiotherapy (DART) consists of the following steps:

1. Acquisition of dose fluence matrices with the onboard EPID system:

The dose fluence matrix was measured with the electronic portal imaging device (EPID), product name $i_{V i e w}{ }^{R}$, of an Elekta Infinity ${ }^{\mathrm{R}}$ Linear Accelerator. To accomplish this, a method was developed for calibration of the dose response of the EPID so that a given gray level of the image represents a given dose level. A calibration curve was established using a sequence of increasing dose levels and signal response measurements by the EPID. Furthermore, in-air scatter contributions and scatter from within the housing of the EPID was measured and the EPID dose response calibration curve was adjusted accordingly.

Subsequently, the delivered dose fluence for each beam was measured by the EPID. 


\section{Cureus}

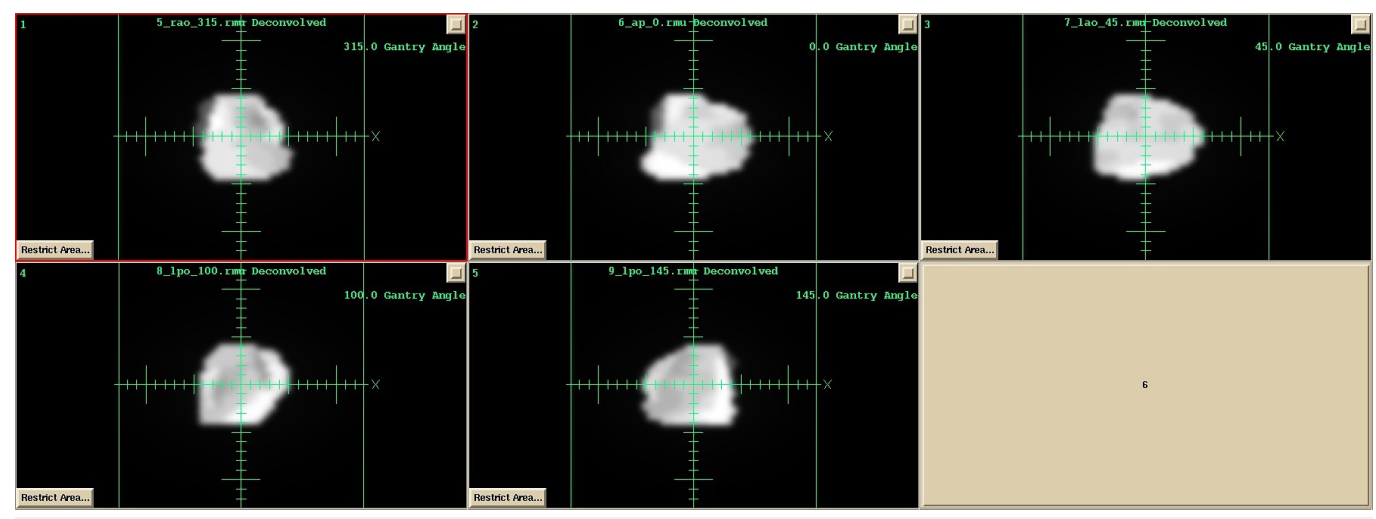

FIGURE 1: Dose fluence matrices for treatment fields. Image brightness is a measure of dose level.

2. CBCT acquisition of the patient anatomy information with the patient in the treatment position:

The daily kV CBCT system of the Elekta Infinity Linear Accelerator, product name XVI ${ }^{\mathrm{R}}$, was utilized to acquire image data sets with the patient in the treatment position prior to the delivery of treatment. The RT structure set generated during the treatment planning process was overlaid for better visualization and subsequent dose evaluation.

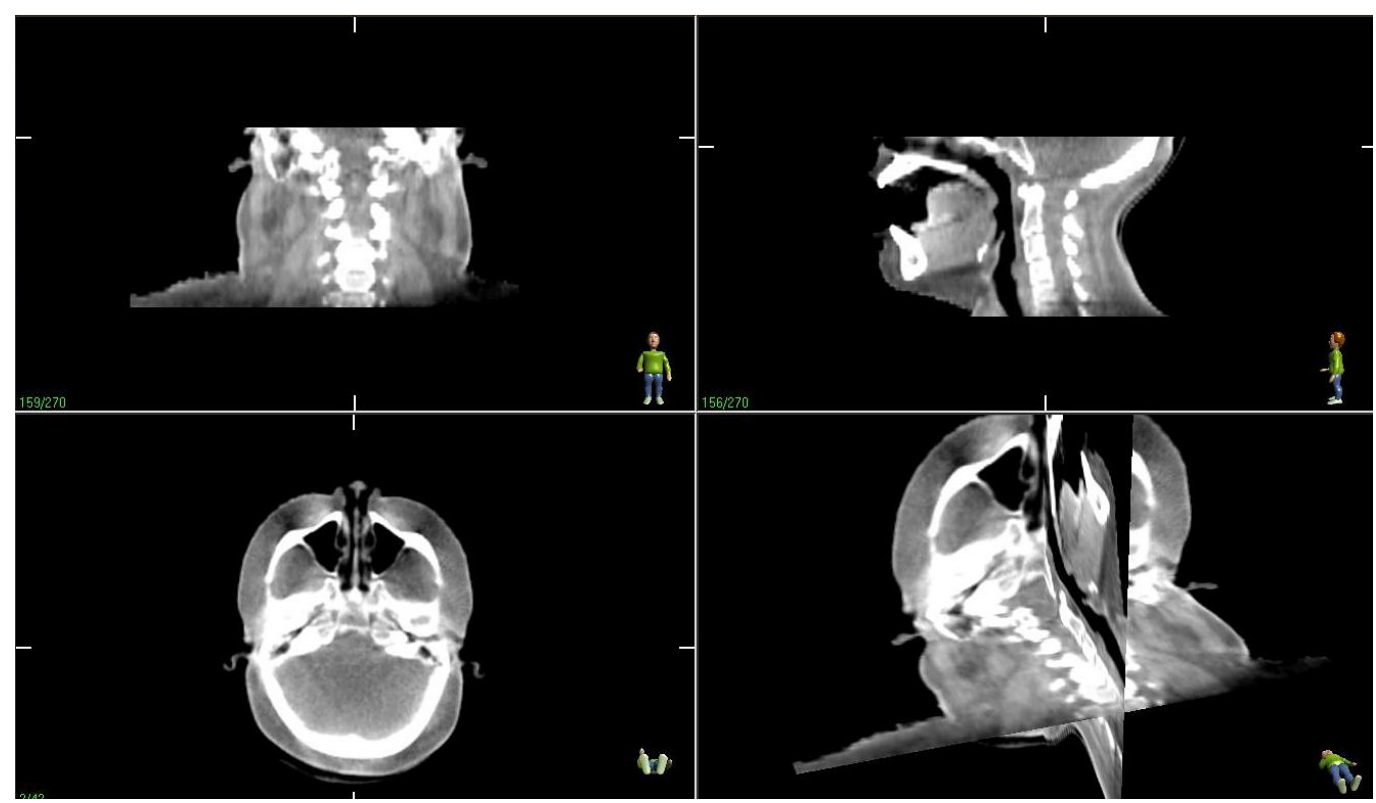

FIGURE 2: CBCT image data set, reconstructed in the three major planes, axial, sagittal, and coronal. PTV and critical structures are embedded in and registered to this image data set.

3. Deformable registration of original image data set to the CBCT image data set: 


\section{Cureus}

The original image data set acquired during the CT simulation process with the registered anatomical structures was fused to the new image data set acquired during the CBCT imaging process with the patient in the treatment position. This fusion was accomplished by using Raystation ${ }^{\mathrm{R}}$ software developed by Raysearch. Rather than applying a rigid fusion, the original image data set was registered to the new data set using deformable registration. A threedimensional deformation matrix consisting of directional vector elements, assigned to each of the image voxels, was established. This matrix was applied to the anatomical structure sets as well. As the original CT image data set is deformed, fused to the CBCT image data set, and remains the primary image data set used for dose calculation, the electron density representation of the CBCT is not relevant to the dose calculation and will not need to be adjusted.

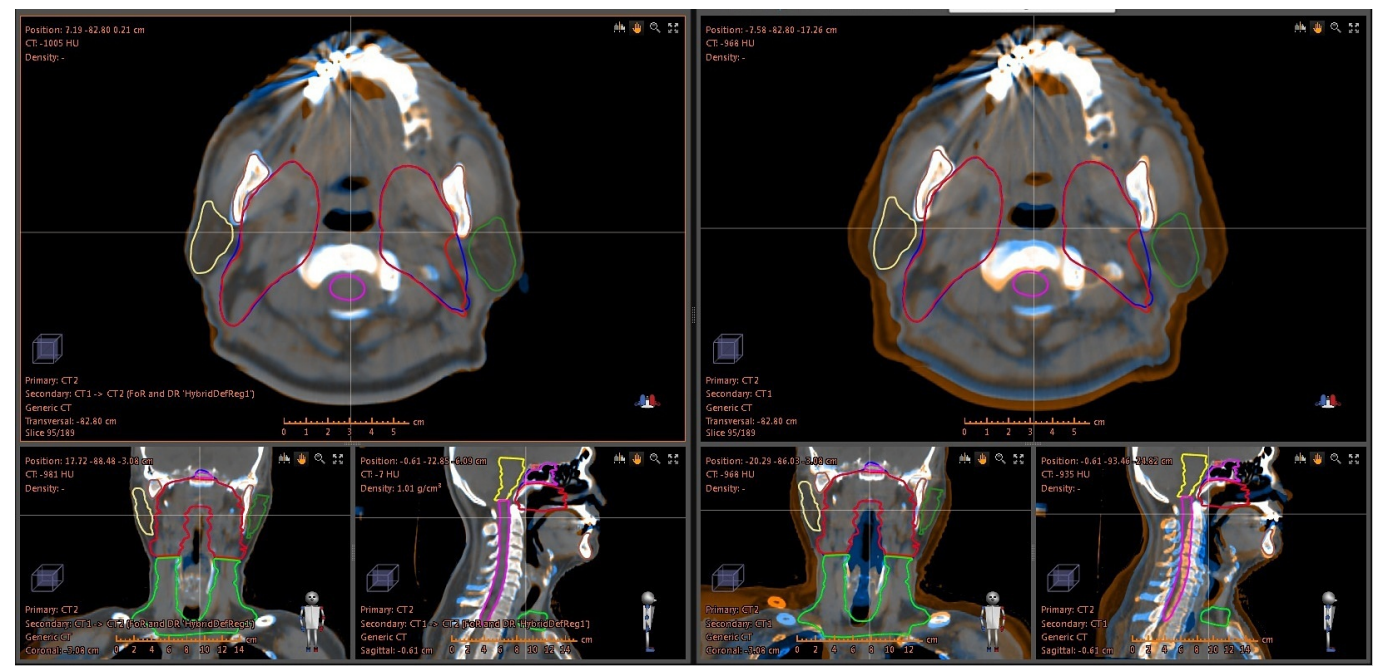

FIGURE 3: The right side shows the original image data set rigidly fused to the current image data set. The left side shows the deformed registration of the original image to the new image data set.

4. Calculation of dose from the fluence matrix as applied to CBCT image data and the generation of isodose distributions:

The acquired dose fluence was applied to the deformed original CT image data set and the dose was calculated by applying a convolution algorithm for each of the beams from the specific gantry angles. The dose was normalized to its maximum dose point and was represented by isodose lines, connecting points of identical dose. The calculation was accomplished by use of Dosimetry Check $^{\mathrm{R}}$, a MathResolutions product. 


\section{Cureus}

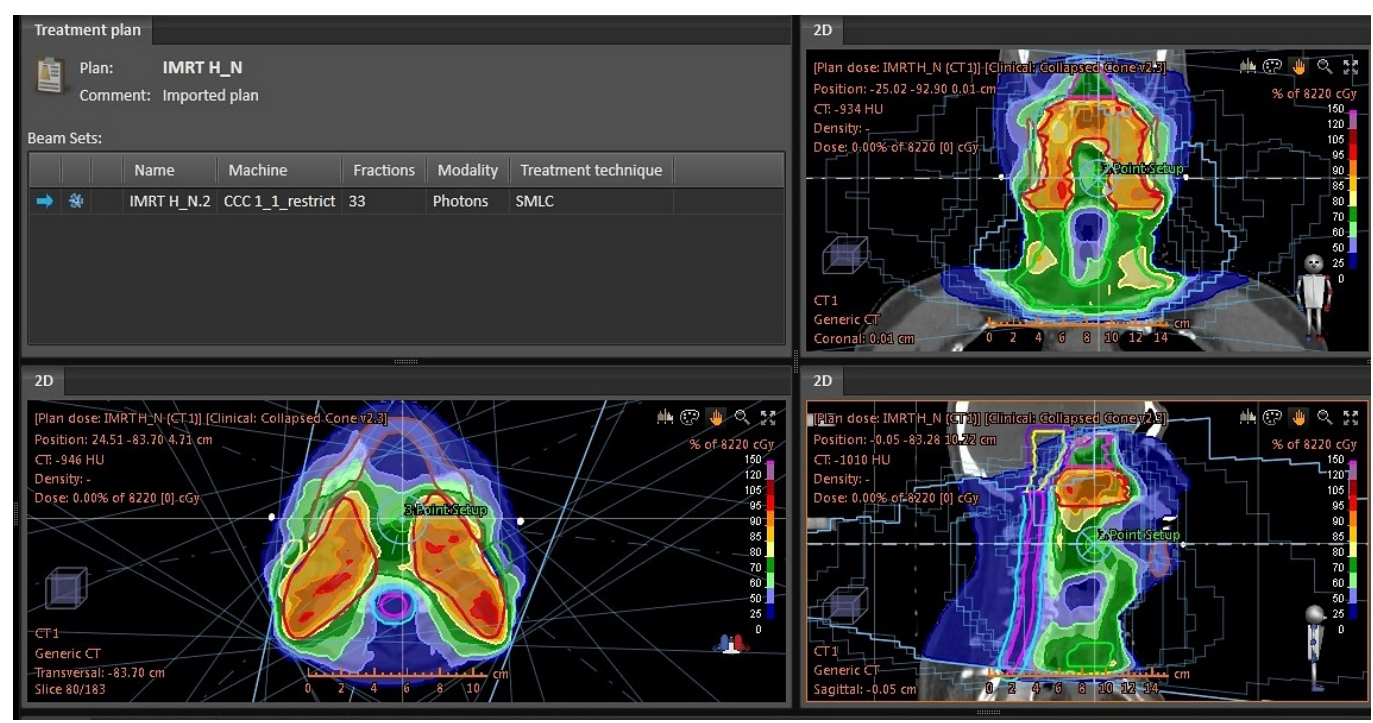

FIGURE 4: Isodose distributions of the calculated dose based on deformed patient anatomy and known dose fluence.

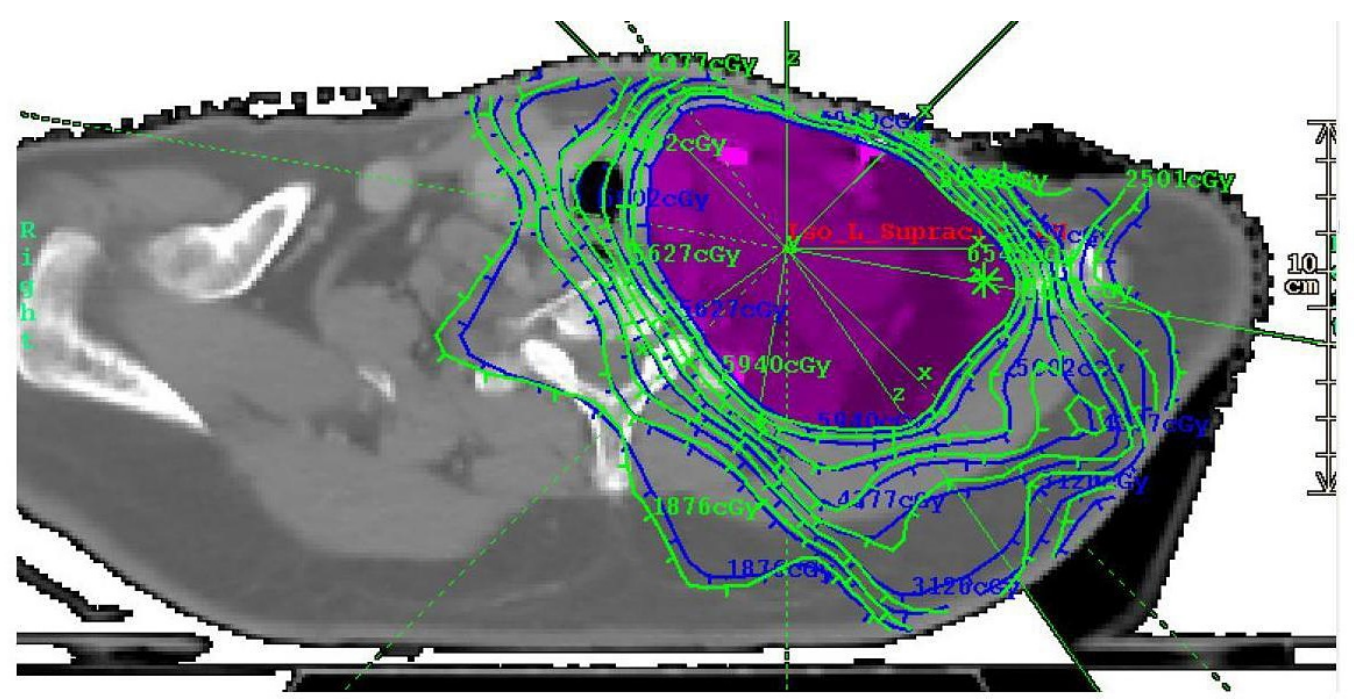

FIGURE 5: Dose comparison of recomputed dose vs. treatment plan dose in the axial plane. Original isodose distribution is shown in green, the recalculated distribution is shown in magenta. 


\section{Cureus}

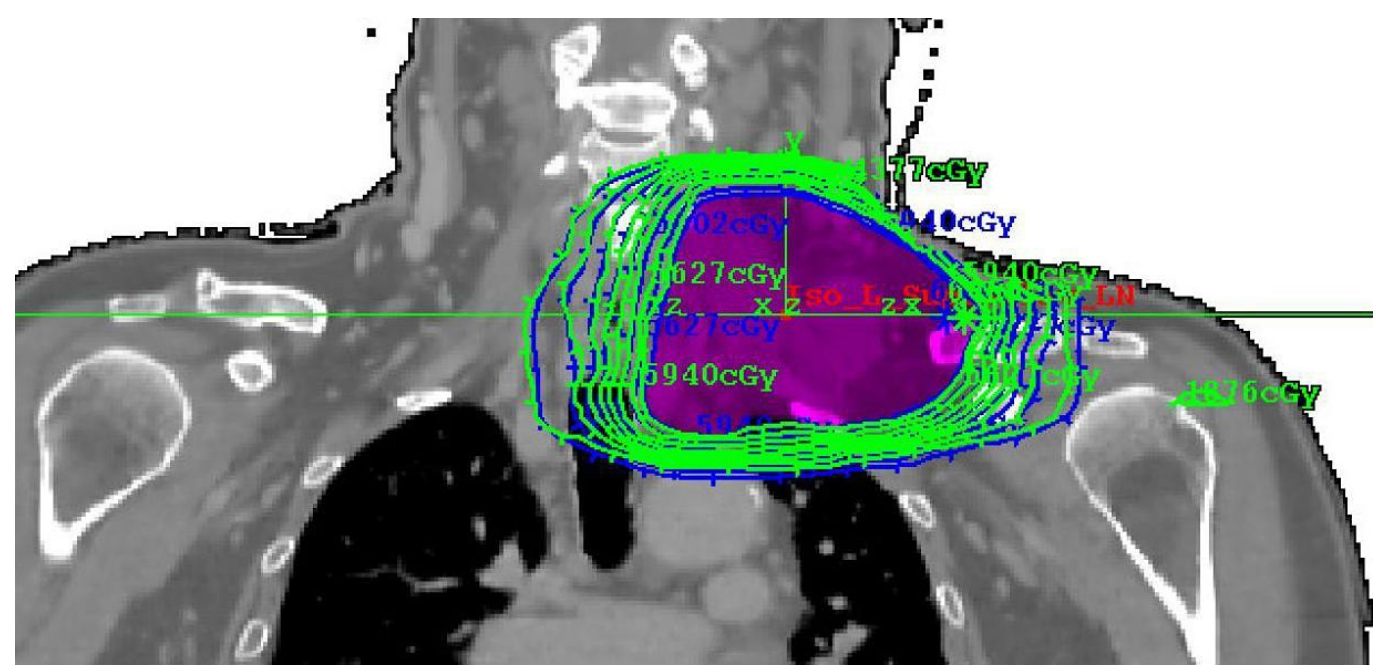

FIGURE 6: Dose comparison of recomputed dose vs. treatment plan dose in the coronal plane.

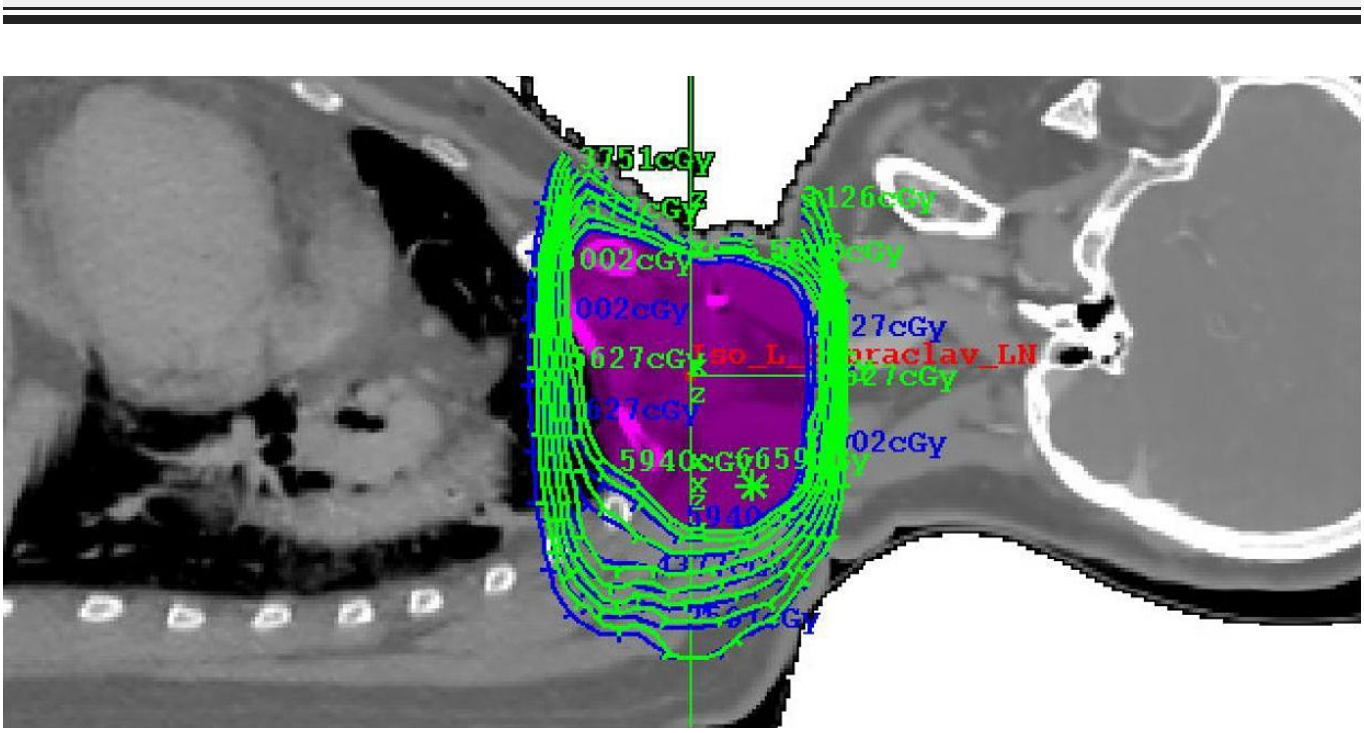

FIGURE 7: Dose comparison of recomputed dose vs. treatment plan dose in the sagittal plane.

\section{Calculation of DVH for target volumes and critical anatomical structures:}

As the contours for target and critical anatomical structures are registered to the CBCT image data set, the dose that is anticipated to be delivered to the patient is now computed based on the new patient anatomy. For this purpose, dose volume histograms were generated for the deformed image data set target volume and each anatomical structure. These dose volume histogram curves were compared with those from the treatment plan.

A volumetric gamma analysis was performed to evaluate quantitatively the dosimetric and spatial variation of the new dose distribution compared to the original dose distribution. 


\section{Cureus}

Dose Volume Histogram (dotted is Reference Dose). Reference Dose: TPS L_Supraclav_LN

Magnification unknown.

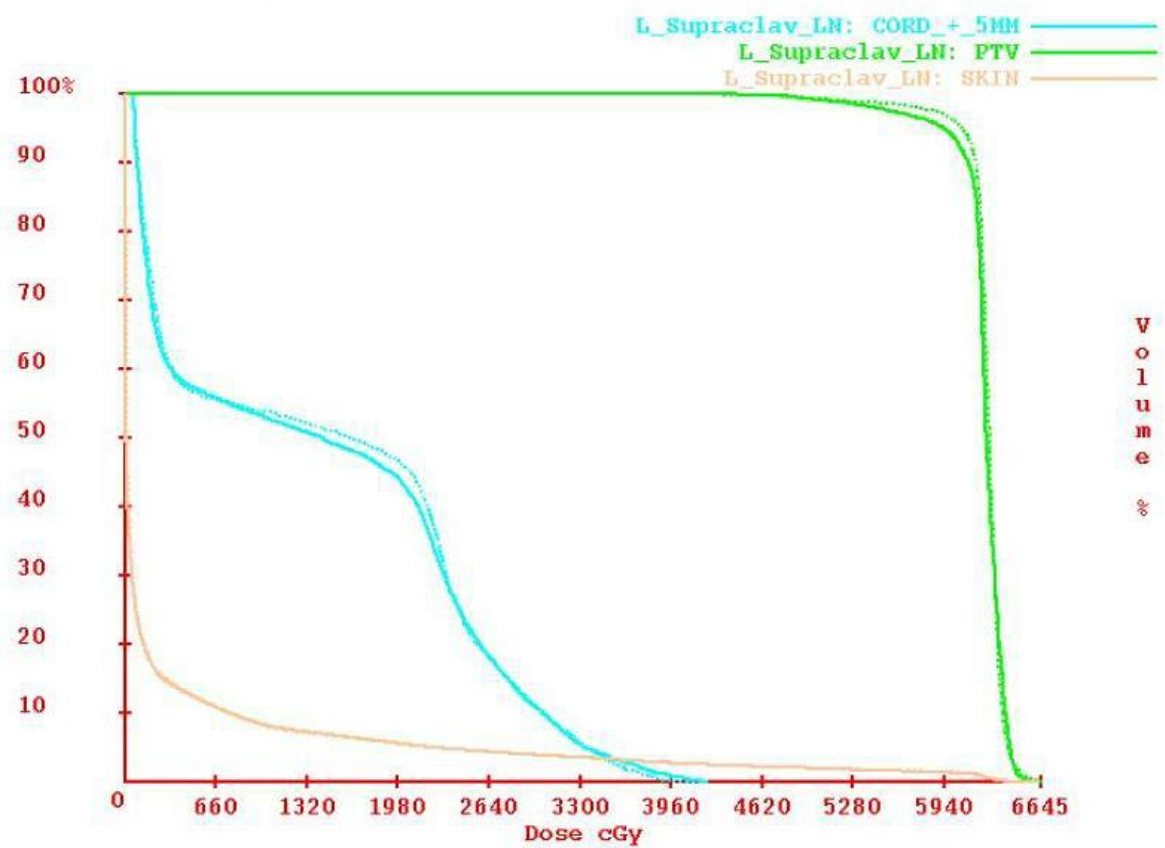

FIGURE 8: Dose volume histogram curves for the original plan (dashed curves) and the new recalculated dose (solid curves). DVH curves for the entire patient, PTV, and spinal cord. 


\section{Cureus}

Gamma Volume Histogram. Reference Dose: TPS L_Supraclav_LN

Magnification unknown.

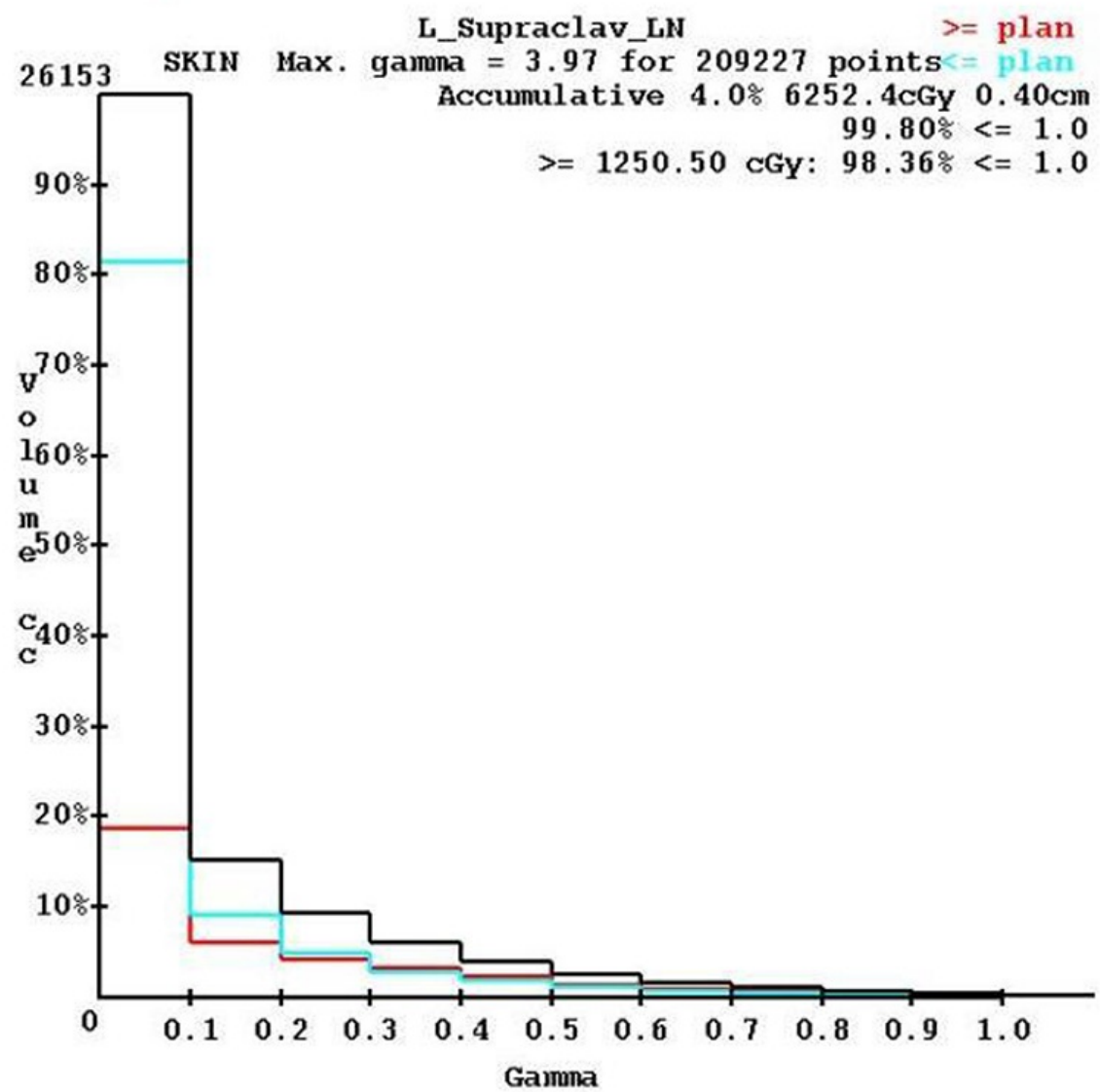

FIGURE 9: Gamma analysis for the entire patient anatomy.

6. Adjustments of the original treatment plan to compensate for the anatomical changes discovered during this process:

A preliminary system of dose-guidance decision support tools was developed that will allow the user in real-time to evaluate dosimetrically the validity of the imminent treatment delivery and to make adjustments to the dose fluence matrix in real-time.

At our clinic, we established criteria that would trigger re-optimization of a given treatment plan:

a.) a dose deviation of $5 \%$ or more at any given point,

b.) a loss of volumetric target coverage of $5 \%$ or more,

c.) a spatial deviation of a given dose by $4 \mathrm{~mm}$ or more.

\section{Results}

Measurement of the IMRT dose fluence matrices and its convolution with the $\mathrm{kV}$ CBCT acquired with the patient in the treatment position allows to quantitatively evaluate the dose distribution and dose volume histograms for the target volumes and critical anatomical 
structures. The effectiveness and impact of the imminent treatment can be evaluated and the treatment can be accepted or adjustments to the dose fluences made. Such dose-guided adaptive radiation therapy (DART) can be accomplished online with the patient awaiting treatment. This system provides the radiation oncology clinical team the tools to predict the dose delivered before each individual treatment fraction and to adjust the treatment plan to reproduce the dose distribution of the original treatment plan and meet clinical treatment objectives.

\section{Discussion}

All intensity-modulated radiotherapy (IMRT) treatments could be improved by the presented method of DART; however, specifically, patients receiving complex IMRT treatments, those who are experiencing anatomical changes during their treatment course, receiving treatment plans with very steep dose gradients, stereotactic ablative body radiation therapy (SABR), and those patients receiving hypo-fractioned treatments will benefit. At our institution, approximately 300 patients per year can be considered candidates for this new clinical method. Of the 2.2 million cancer patients per year treated worldwide with radiotherapy [11], 15\% or 330,000 patients are expected to receive the type of treatment that would benefit from the described process.

In order to provide this system as a real-time tool at the treatment console, several technical, staffing, and process requirements will have to be met. Computing power will need to be fast enough to deform the reference image data set onto the day of treatment CBCT data set, apply the dose fluences to each of the fields, and compute the dose and the gamma evaluation. The level of education and training for the treating therapists will need to be at a level where clinical online evaluations and decisions can be made to accept the existing treatment plan or reject it to make required adaptations. Lastly, the clinical process will need to be established that will allow the additional time needed for this process. A possible practical solution might be to perform this process once a week and implement any necessary plan adaptations with the following treatment fraction.

It is estimated that the total treatment time for a head and neck treatment will be $20-25 \mathrm{~min}$. If a simple dose scaling of the evaluated plan is required, a recomputation of monitor units can be accomplished with the patient in the treatment position. If a new plan needs to be generated, this process will be performed prior to the next treatment fraction.

\section{Conclusions}

Radiation oncology will benefit from online DART by providing a tool to the clinical team for the evaluation of treatment fractions individually, adjust the plan to meet clinical needs, and to document the delivered dose. DART allows better verification and assurance of dose delivery. Treatments will be delivered with a higher degree of accuracy; therefore, the treatment prescription doses can be escalated while maintaining dose sparing of nearby critical structures. It will be possible to increase the complexity of treatment plans with steeper dose gradients and more customized dose distributions with confidence. Consequently, cure rates for this type of treatment are statistically expected to increase, while occurrence of complications and toxicities are expected to decrease for the same given dose.

\section{Additional Information}

\section{Disclosures}

Human subjects: All authors have confirmed that this study did not involve human participants or tissue. Animal subjects: All authors have confirmed that this study did not involve animal subjects or tissue. Conflicts of interest: In compliance with the ICMJE uniform 
disclosure form, all authors declare the following: Payment/services info: All authors have declared that no financial support was received from any organization for the submitted work. Financial relationships: All authors have declared that they have no financial relationships at present or within the previous three years with any organizations that might have an interest in the submitted work. Intellectual property info: Two patents are held by G. Weidlich in the field of Dynamic Radiotherapy. Other relationships: All authors have declared that there are no other relationships or activities that could appear to have influenced the submitted work.

\section{References}

1. Yan D, Lockman D, Brabbins D, Tyburski L, Martinez A: An off-line strategy for constructing a patient-specific planning target volume in adaptive treatment process for prostate cancer. Int J Radiat Oncol Biol Phys. 2000, 48:289-302.

2. de la Zerda A, Armbruster B, Xing L: Formulating adaptive radiation therapy (ART) treatment planning into a closed-loop control framework. Phys Med Biol. 2007, 52:4137-53.

3. Wu C, Jeraj R, Olivera GH, Mackie TR: Re-optimization in adaptive radiotherapy. Phys Med Biol. 2002, 47:3181-95.

4. Yan D, Lockman D, Martinez A, Wong J, Brabbins D, Vicini F, Liang J, Kestin L: Computed tomography guided management of interfractional patient variation. Semin Radiat Oncol . 2005, 15:168-179.

5. Court LE, Dong L, Lee AK, et al.: An automatic CT-guided adaptive radiation therapy technique by online modification of multileaf collimator leaf positions for prostate cancer. Int J Radiat Oncol Biol Phys. 2005, 62:154-63.

6. Rijkhorst EJ, van Herk M, Lebesque JV, Sonke JJ: Strategy for online correction of rotational organ motion for intensity-modulated radiotherapy of prostate cancer. Int J Radiat Oncol Biol Phys. 2007, 69:1608-17.

7. Wu Q, Liang J, Yan D: Application of dose compensation in image-guided radiotherapy of prostate cancer. Phys Med Biol. 2006, 51:1405-19.

8. Birkner M, Yan D, Alber M, Liang J, Nüsslin F: Adapting inverse planning to patient and organ geometrical variation: Algorithm and implementation. Med Phys. 2003, 30:2822-31.

9. Löf J, Lind BK, Brahme A: An adaptive control algorithm for optimization of intensity modulated radiotherapy considering uncertainties in beam profiles, patient set-up and internal organ motion. Phys Med Biol. 1998, 43:1605-28.

10. Ghilezan M, Yan D, Martinez A: Adaptive radiation therapy for prostate cancer . Semin Radiat Oncol. 2010, 20:130-7. 10.1016/j.semradonc.2009.11.007

11. International Atomic Energy Association (IAEA): Setting up a Radiotherapy Programme: Clinical Medical Physics, Radiation Protection, and Safety Aspects. International Atomic Energy Association. 2008, pp 1-230. Accessed: September, 2012: http://wwwpub.iaea.org/MTCD/publications/PDF/pub1296_web.pdf. 